\title{
A Model for Preliminary Design Procedures of Satellite Systems
}

\author{
Davide Di Domizio and Paolo Gaudenzi* \\ Università di Roma La Sapienza, Dipartimento di Ingegneria Aerospaziale e Astronautica \\ Via Eudossiana 18, 00184 Rome, Italy
}

\begin{abstract}
The article describes a model for satellite systems preliminary design based on European Space Agency concurrent design approach. Space systems preliminary design aspects based on a concurrent engineering methodology are first briefly illustrated. A general description of a typical space system and its subsystems is provided and sizing criteria are provided for each subsystem. The design procedure is formalized according to Unified Modeling Language formalism. A description of the implementation of the model and some conclusive remarks are then included.
\end{abstract}

Key Words: Space systems, space sub-systems, concurrent design, system engineering, design procedures.

\section{Introduction}

Since 1998, the European Space Agency (ESA) has been developing preliminary studies for new space missions using the Concurrent Design Facility (CDF) as described in Bandecchi et al. [1, p. 1,2; 2, p. 1-3]. Concurrent Design (CD) is a methodology that allows the parallel design of several subsystems, managing their mutual interactions, which are then assembled to form an engineering system. The use of this methodology is particularly useful in aerospace engineering, where the design is challenged by the presence of very complex engineering systems. ESA has adopted this methodology for the early stage of the design of space systems, following the example of other space agencies, like NASA, and aims to promote the use of this approach, also for more advanced design phases, in the frame of the European Space Industry.

The success of this approach is witnessed by the great reduction of the time needed to produce reports for preliminary analyses of very complex missions - like science or exploration missions - keeping or improving the high level of design and the quality of studies and even facilitating the communications between designers and customers. In ESA, the duration of a pre-Phase A analysis is now between 3 and 6 weeks against 6-9 months about 10 years ago.

In the context of space engineering the adoption of $\mathrm{CD}$ approaches generated a series of advantages: time savings, reduced errors in sizing, better cost prediction, and a better compliance to mission requirements;

*Author to whom correspondence should be addressed.

E-mail: paolo.gaudenzi@uniromal.it

Figures 1-7 appear in color online: http://cer.sagepub.com however, the performances of the CDF are the most interesting aspect. The success of CDF approach dwells in the cutback of the time to produce a pre-Phase A study. The space system is divided into several subsystems assigned to specialized engineering units which manage the design of each part; obviously the design of each subsystem is strictly correlated with the others: an information flux is necessary. In these preliminary phases, each subsystem can be characterized by a series of 'design parameter', which describe its most relevant physical and technical properties. Design parameters are the information exchanged during CDF session among engineering units.

This design approach requires hardware and software resources, as implemented by ESA in the CDF releases in the European Space Research and Technology Centre, but before that a clear description of the system and of the sizing and dimensioning criteria has to be adopted for the overall system, for the payload, and for each bus subsystem. Since the design develops in a concurrent framework, all the interactions among subsystems are required to be well documented too. An effective data modeling will produce clear interactions during the design phases and an easier and more efficient exchange of information [3, p. 2000], solving the problems encountered in data transfer among different areas, as already discussed in [4]. In addition, it furnishes a successful and tested answer to meta-data description as requested in [5], improving team coordination in contexts where a different framework is already applied [6].

The aim of the present article is to describe the model for satellite systems preliminary design developed at Universita di Roma La Sapienza in the frame of the professional Master Course in satellites and based on 
ESA concurrent design approach. The study is focused on radar Earth Observation missions. In fact there is the need to focus on a certain class of satellite systems with well-defined requirements to obtain a formal complete description of the model for preliminary design. However, this has not to be considered as a limitation to the generality of the present work illustrated which is focused on the procedure followed during the design. Therefore, a general description of the design and of the design variables is provided along with their mutual interactions in the dimensioning and sizing schemes provided for each subsystem and implemented according to Unified Modeling Language (UML) formalism.

In the following paragraphs all the steps followed to accomplish the design are described. First of all, the common approach to space systems design is briefly illustrated, followed by a slight description of the case study use as reference in this work. A concise explanation of the instruments used to document the design procedure is then provided. The section entitled 'Modelling the design procedure with UML diagrams' represents the core of the present effort: each diagram produced in the design phase is shown and described. A description of the implementation of the model and some conclusive remarks are then included.

\section{The Pre-Phase A Design Process of a Satellite System}

A space mission can be described in terms of space, ground and launch segment [7]. The space segment (the spacecraft) consists of a payload and a bus, composed by several subsystems. Payload is the set of instruments used to meet the objective of the mission: observing the Earth, broadcasting communications, or analyze the atmosphere. The bus has several aims concerning with the functionality, operating survival, and the efficiency of the payload in the space environmental conditions.

Ground segment must interact from ground with the spacecraft both for the payload and for the bus purposes and is responsible of actions like:

- Receiving and analyzing the telemetries of the spacecraft to monitor its operating status

- Send commands to the spacecraft when needed

- Receive payload data transmitted from the spacecraft or, conversely, transmit data to the spacecraft payload.

The ground segment also operates during the launch phase, a very critical phase of the spacecraft life.

The activities of the launch segment are related to the phases of a space mission that safely takes the spacecraft from the earth surface to the final orbit. The space transportation system is very complex and comprehends both the launch vehicle and the launch basis, connected with the ground segment.

The procedure proposed in this work is focused on the preliminary design of the space segment.

During the first stage of the process of spacecraft design, mission team analyzes mission requirements and produces system and payload constraints for system and payload specialists. These requirements regard the choice of orbit properties, the lifetime, operations, and the main features of the instruments (e.g., resolution or revisiting time in earth observation or link budget in communication). Clearly, this first step establishes a series of requirements and goals to be met for the payload and also sets the operational conditions to be performed by the bus, to allow the mission to be performed in an effective way.

A very synthetic description for the classes of functions to be performed by the bus can be illustrated as follows:

- Support the payload mass and all the masses onboard the spacecraft

- Provide the propulsion needed for orbital manoeuvres and corrections

- Provide attitude control to the spacecraft and point the payload correctly

- Keep the payload (and the entire spacecraft) at the proper temperature conditions

- Provide electric power

- Provide on board data storage and elaboration

- Establish a link to ground for transmitting telemetry and receiving commands

It is usual in space system engineering to associate these functions to one subsystem of the spacecraft [7]. A typical list of subsystems can be defined as follows: payload, Attitude and Orbital control (AOCS), structure, propulsion, data handling, thermal, power, Telemetry Tracking and Command (TT\&C). In general, each subsystem is designed by an engineering unit composed by skilled staffs. A system engineering unit should also be considered with the aim of coordinating all the others, managing the system budgets (e.g., mass, power, link) and respecting the constraints that derive both directly from the requirements or from the mission analysis.

Each engineering unit, corresponding to a subsystem, the payload, or the system itself, has to follow simple sizing rules relevant to a specific discipline. In this effort, optimal values for the design parameter are obtained thanks to basic trade off criteria that most of the time optimize the required performance with respect to weight, power, and link budget.

All the engineering units of the list proposed above also including one Mission unit that is responsible for the characteristics of the mission to be realized - are the main players of the design phases. Each unit aims to design a subsystem, characterizing it by a series of 
design parameters: factors describing a specific feature of the unit. At the end of the design process a specific value will be assigned to all design parameters. Examples for design parameters are the following.

- System: Dry mass of the spacecraft, Mass of each subsystem, etc.

- Payload: Band of the Synthetic Aperture Radar (SAR) Antenna, Digital data rate of acquired data, etc.

- Structure: Structural mass, Body height, etc.

Some parameters belong entirely to a single subsystem and are used only for internal evaluations of subsystem design; other design parameters, since a concurrent logic is assumed, are typically exchanged among subsystems: the computations of one subsystem are the input for the analysis of features of another one. (e.g., The design parameter digital data rate produced by payload is an input for data handling which has the aim to store data coming from payload).

The present model of satellite design aims to describe the interaction among the design units and allows a contextual evaluation of the effects that a change of a parameter adopted in a single subsystem could produce in another one. As an example, if the number or locations of batteries are modified, then the structural system needs to update its structural loading requirements while AOCS has to verify the new centre of gravity position and the new inertial rigid body characteristics. Moreover, for both units, a new value for the design parameters (and relevant geometries, material, component) could be necessary. The number of the design parameters, limited at a preliminary phase of the project, is assumed to grow along with the level of detail of the project.

As stated in ECSS publications ${ }^{1}[8]$, from the Mission analysis (Phase 0) to Feasibility (Phase A) there are different aims to be satisfied, therefore there is a requirement for more details that is directly translated in a higher number of design parameters. The increasing need for more detail is also characteristic in the next two phases (B and C): preliminary definition and detailed definition. In the present article the application of the CD approach is limited to the first two phases (prePhase A study); however, the procedure described in this article could be valid to describe the relations among the components of a single subsystem when the design has entered more specific stages.

In the present work, the design procedure described before is implemented for to the pre-Phase A design of a spacecraft and applied to the specific space mission proposed in the next paragraph. At this preliminary design level it is important to choose the set of design parameters that, at the same time, are limited in number but effective in describing the system at the level of detail needed.

In the modeling effort, a special role is to be assigned to a database of components, where the information collected are the basis for giving a precise value to many design parameters. The efforts in design strategies developed by subsystem designer can have a real confirmation only from the support coming from the properties of existing components. Each designer has access to a database of components relevant for the topics that his subsystem deals with. Each of these databases is based on the following information: company, component type, performance, dimensions, and operational constraints.

\section{Modeling Assumptions and Criteria}

\subsection{Case of Study and Design Strategy}

Although the logic and the modeling procedure could be made very general, in this article an application is presented nearby the design procedure itself. The general method has been applied to the design of an Earth Observation Radar satellite. This system is a suitable architecture for a SAR remote sensing satellite mission, to be operated in a Sun synchronous orbit. The orbit requested was Sun Synchronous at $619 \mathrm{~km}$ and $97.87^{\circ}$.

Table $1[9$, p. iii] summarizes the main requirements of the mission - the focus of design example. The design methodology takes advantage of teamwork activities performed during the editions of Master Course in Satellites of the Università di Roma La Sapienza.

Although the choices made during this example are very specific and lead to a precise design output, the logic and the modeling procedure could be considered as very general. Specifically, the Earth Observation (EO) space system has the aim to retrieve images and send them to the users following the requirements stated above, but while the design phase of each subsystem is oriented to achieve this aim, the design procedure is quite independent from the actual system, and could be generalized to other type of missions.

As described previously, the satellite in the example is divided in to several units and a specific engineering unit is assumed to be present. The complete list of units is: mission, system, payload, structure and configuration, power, AOCS, propulsion, thermal, TT\&C, data handling, payload-data handling, and transmission.

The strategy that is followed during the various phases of the preliminary design is managed by the

\footnotetext{
${ }^{1}$ ECSS (European Cooperation for Space Standardization) Standards documents are intended to be applied together for the management, engineering and product assurance in space projects and applications. ECSS is a cooperative effort of the European Space Agency, National Space Agencies, and European industry associations for the purpose of developing and maintaining common standards.
} 
Table 1. Mission Requirements for the required satellite system.

\begin{tabular}{llll}
\hline Description & \multicolumn{1}{c}{ Values } & \multicolumn{1}{c}{ Description } & Values \\
\hline Min observation angle & $20^{\circ}$ & Bus voltage min/max & $23 / 38 \mathrm{~V}$ \\
Max observation angle & $50^{\circ}$ & PDHT downlink frequency & $8.1 \mathrm{GHz}$ \\
Ground resolution & $3 \mathrm{~m}$ & Pointing accuracy & $0.0028^{\circ}$ \\
Image mode & STRIPMAP & TT\&C uplink frequency & $2050 \mathrm{MHz}$ \\
SAR frequency & $9.65 \mathrm{GHz}$ & Downlink data rate & $155 \mathrm{Mbps}$ \\
Minimum swath & $40 \mathrm{Km}$ & SAR duty cycle & $10 \%$ \\
Min no. images per day & $>50$ & Operative life & 5 years \\
Polarization & $\mathrm{HH}, \mathrm{VV}, \mathrm{HV}, \mathrm{VH}$ & Revisit time & $\sim$ Day \\
CPU architecture & Integrated & Operative autonomy & $24 \mathrm{~h}$ \\
\hline
\end{tabular}

system designer that has the principal aim to respect the requirement on the driving budgets. ${ }^{2}$

Therefore, budgets are the summary descriptors of the entire system and usually can consist of mass, power, telemetry, propellant, pointing, or reliability budget. Mass budget is the most effective driving budget, because it directly influences the mission costs and is the one that influenced the example presented in this article. During the steps of the analysis, the system designer controls the mass values and the mass evolution of the entire system and of each subsystem. The design is assumed to be compliant with the requirements on mass budget when system and subsystems mass are stable and further iteration steps will not modify their values. In the case study, a name was assigned to a possible satellite complying with mission requirements: FINGERSAT (First Idea of a New Generation Earth observation Remote sensing Satellite).

\subsection{UML Methodology and Diagrams}

The documentation of the design procedure is a necessary support to the analysis describing the interactions between subsystems and the activities performed by designer during the design sessions. This documentation uses UML formalism, implementing static, collaboration and activity diagrams $[10,11]$. In this article, UML diagrams are then used to describe the Concurrent Design approach to the preliminary analysis of a spacecraft.

A class diagram describes the types of objects ${ }^{3}$ in the system and the various kinds of static relationships that exist among them. Class diagrams typically show the properties (attributes) and operations (methods) of a class and the constraints that apply to the way objects are connected (associations). The relationships among classes may be of several types: generalization, aggregation, composition and generic associations.

In the next paragraph, each subsystem is documented by a specific class diagram and occasionally some functions are detailed by activity diagrams, while the interactions among subsystems are depicted in collaboration diagrams. The structure of class diagrams is fixed in this work. The central part of the diagram contains a class representing the subsystem that is intended to be described (payload in Figure 3). This is the general part of the subsystem; it may have some attributes and methods. Below this main class there are several other classes that are linked to it with associations one-to-one. These classes, named with the stereotype "data exchange ${ }^{4}$ represent the part of the subsystem that exchanges values with other subsystems - the interface to other subsystems. Each main class may have several 'data exchange' classes, one for each subsystem that has to exchange data with (I.E. 'Payload_Power' interface class contains attributes that payload shares with power subsystem and methods that permits payload to evaluate these attributes - Figure 3).

A collaboration diagram (Figure 6) is a special class diagram that shows what happens to the objects when they are instantiated. ${ }^{5}$ The links between entities represent messages, data, or actions exchanged among them.

Activity diagrams are a technique to describe procedural logic, business process, and work flow. In many ways, they play a role similar to flowcharts, but the principal difference between them and flowchart notation is that they support parallel actions.

\section{Modeling the Design Procedure with UML Diagrams}

In this paragraph, the most relevant diagrams of the design procedure are shown: their structure and organization are a feature of the procedure while the data contained depends on the example mission considered in this work.

All class diagrams are presented: one for each subsystem. This set gives a complete description of the

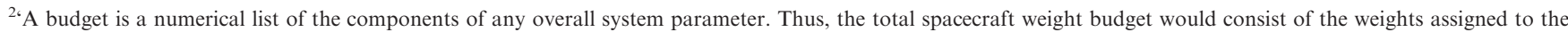
payload instruments, the various subsystems, the propellant required, and typically some margin for growth' (4, paragraph 10.3).

${ }^{3}$ Objects and classes are part of the common idiom used in OO-design, it can be easily translated to other fields of applications: in the Figure 1 the class 'Payload Component' is though as the classifier for all the components of the payload (objects): antennas, RF components, cables, etc.

${ }^{4} \mathrm{~A}$ stereotype is a further description of a particular set of classes.

${ }^{5}$ In the present case, the instantiation of a class occurs during the CDF session when the data are really exchanged among subsystems.
} 
static view of the entire system. Two examples of dynamic diagram are reported.

A collaboration diagram describes the sequence of the activities and the involved subsystem of the data handling engineering unit. An activity diagram depicts the detailed procedure used by payload data handling and transmission for mass memory sizing.

The dynamic diagrams presented work at a different level: the collaboration one is an inter - subsystem document diagram, while the activity diagram is an intra subsystem diagram.

\subsection{Mission}

The mission analysis engineering unit represents the part of the system that make the first analysis and translates the system requirements, shown in Table 1, in specific requirements for each subsystems. Therefore, in this diagram (Figure 1) there are not input labels because Missions generates only outputs and basically is not receiving inputs from other units.

\subsection{System}

The diagram that describes the activities of system designer is quite composite as it has to show the interactions with all the other subsystems (Figure 2). Therefore, the principal feature of this diagram is the presence of interfaces with all other subsystems. The common data that all subsystems exchange with the system designer is the mass budget. The system designer gives a mass budget to each subsystem, the latter has to develop the design respecting this constraint, and at the end of each CD step, gives the results to system designer in terms of mass budget and eventually mass margin. Another common datum that system engineering unit receives from all subsystems is estimated the estimated power need.

Besides, there are more specific data that characterize the interaction with other subsystems. In this domain, system gives to payload engineering unit information on pointing accuracy and on the range of power that can be furnished, while it passes to structure basic mass properties; it gives to data handling information on ground station and on the telemetry budget, to TT\&C the requirements on error rates, and to propulsion the data on propellant mass. Power engineering unit receives from system the requirements on bus properties while AOCS gets some pointing requirements. Finally, thermal needs specific values of dissipated power on the various operating modes of the spacecraft.

The inputs to system are essentially mass budgets data except for mission that gives some information on the launcher and the chosen ground stations. Other more specific properties are given by other subsystems, as reported in Figure 4.

\subsection{Payload}

The payload engineering unit receives inputs from mission (orbital parameters, requirements on the EO mission) and from system (Budgets and pointing accuracy).

This special kind of subsystem has some characteristics (the name of the apparatus and redundancy

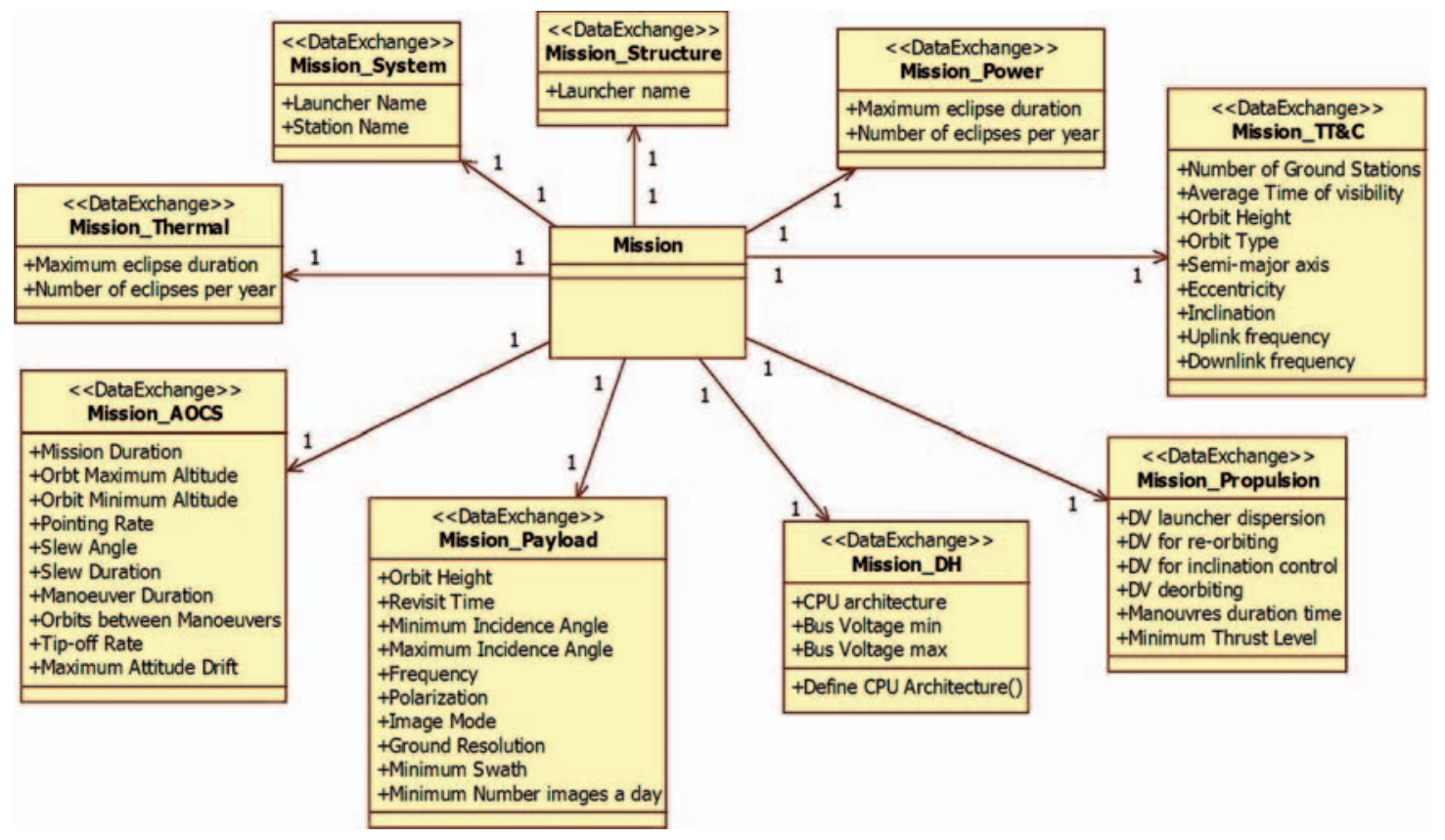

Figure 1. Mission class diagram. 


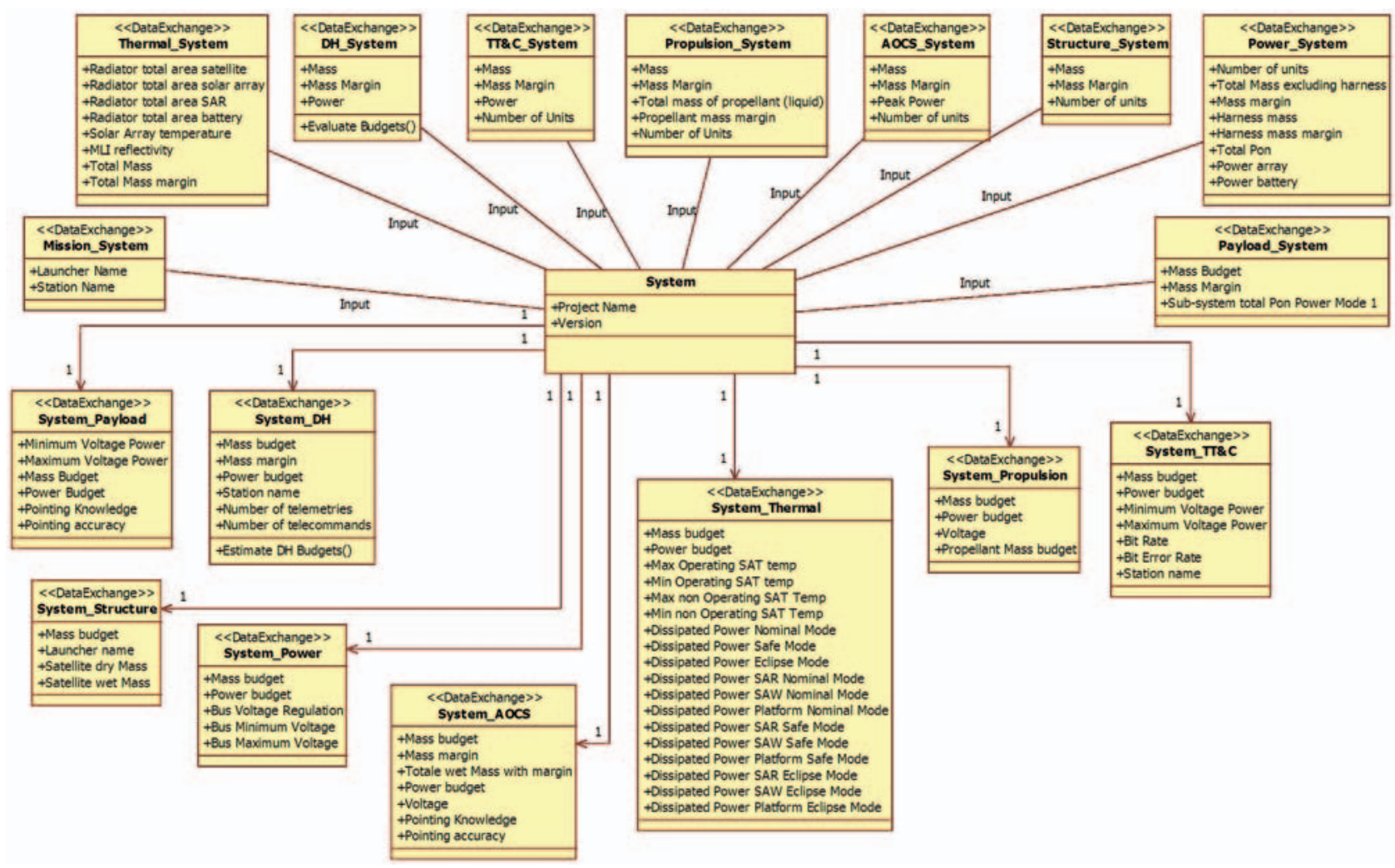

Figure 2. System class diagram.

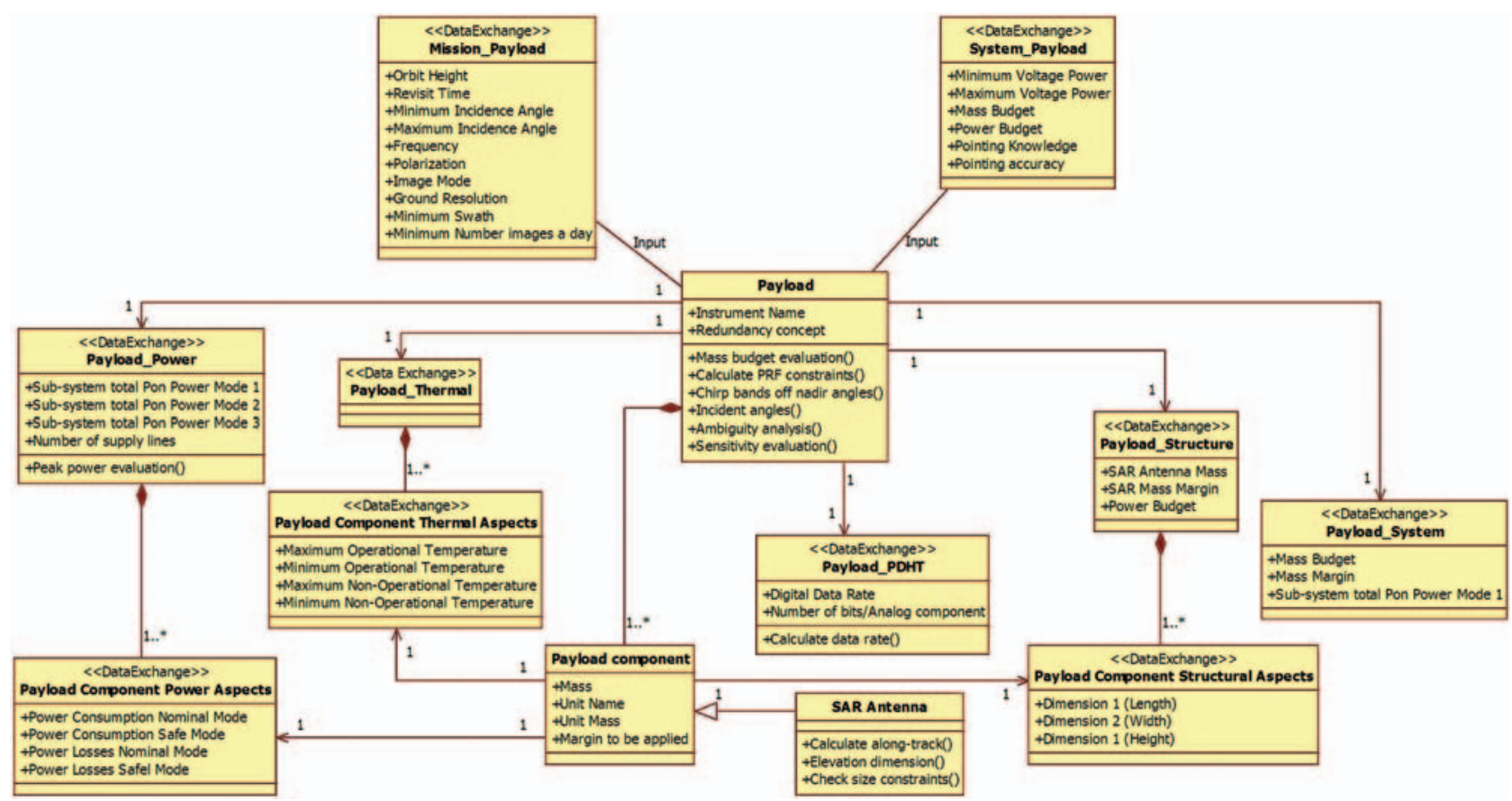

Figure 3. Payload class diagram.

concept) and there are many functions developed to evaluate some instruments features (Figure 3).

Then Payload also outputs many data. To system, as all other subsystems, it gives the payload mass and power budgets; for data handling $(\mathrm{DH})$ it evaluates the data rate needed to collect imagery data, for power it calculates the power supply required by the instruments.

Payload is composed by several components. Each component has some thermal, power, and structural properties: i.e., operational temperatures, dimensions, and masses. 


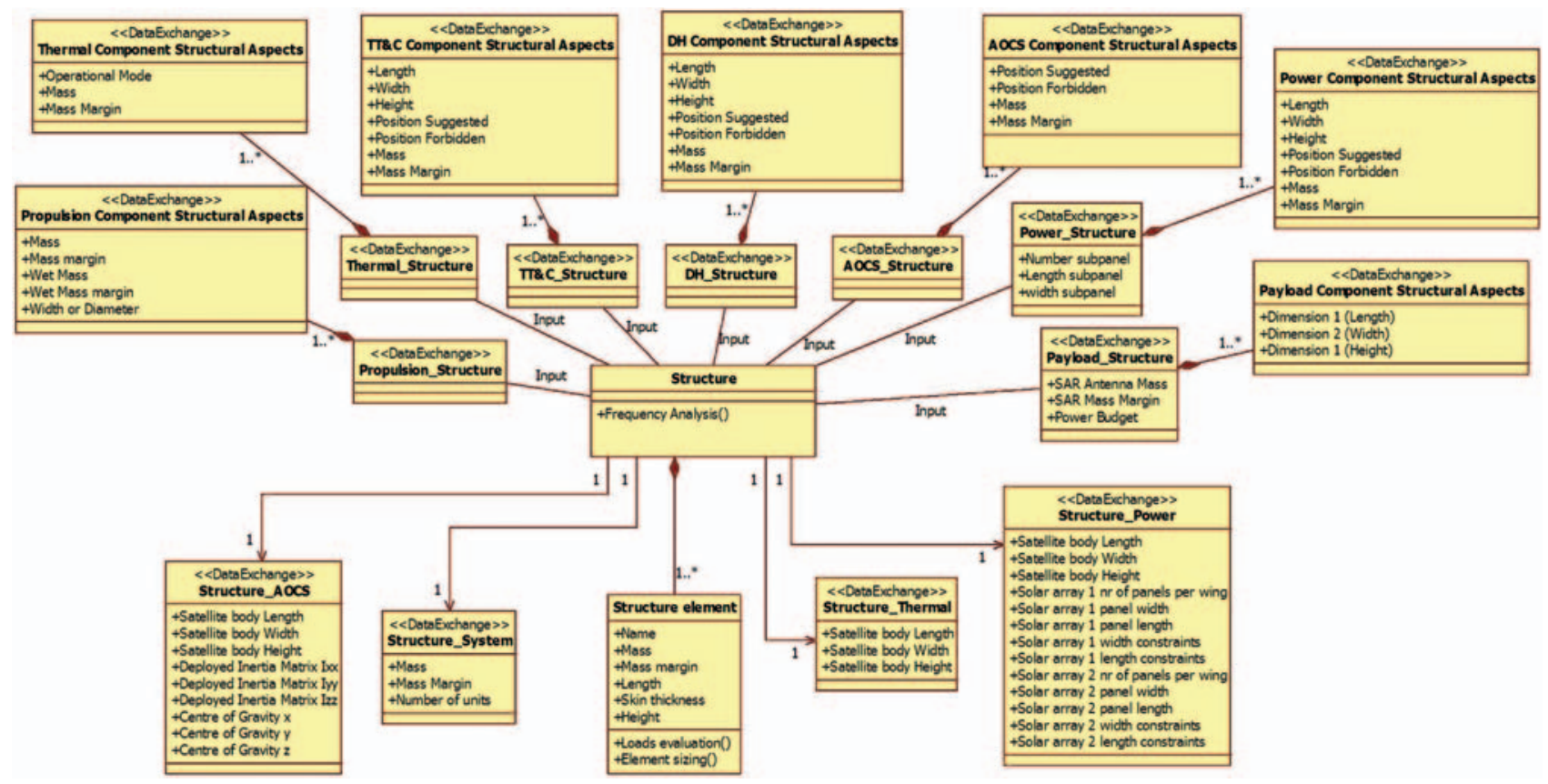

Figure 4. Structure class diagram.

\subsection{Structure}

The structure subsystem unit includes also the configuration aspects, that sometimes are considered as a separate field of expertise for the complexity that the geometry of arrangements and interfaces among all the parts of the spacecraft could reach. This unit receives inputs from all other subsystems. The properties received are in the following category: dimensions, mass, position suggested, and denied during the configuration phase.

Obviously, structure not onley gives information the to system, but also to other subsystems: to AOCS satellite dimensions and its inertia properties, to thermal satellite dimensions and to Power the requirements for the solar wings. Structure is composed by several elements that are designed using the appropriate functions and is described in the corresponding component class (Figure 4).

\subsection{Data Handling}

From the observation of this diagram (Figure 5) it is immediately clear that the data handling subsystem receives the classical data from system engineering unit: mass and power budgets. Besides, some more information is given: the name of the ground station, which data handling will transmit data to, the so-called telemetry budget (that is the number of telecommand and telemetries to be managed by DH subsystem and that will furnish information on the status of all other subsystems during the mission operational phases).

Data handling receives from the mission engineering unit the bus characteristics and the architecture to be used for the processing units. The AOCS features are also essential: frequency and data rate of all sensors drive the choice of processors and buses. In the output area, data handling behaves as other subsystems giving thermal, structural, and power properties of components to the corresponding units (Figure 7).

\subsection{Data Handling Collaboration Diagram}

This diagram (Figure 6) shows the actions when the concurrent design process is in active phase, when the design parameters are exchanged among subsystems, and engineering units make their design choices.

Each arrow indicates a flux of information between objects. The numbers give a possible sequence of the actions. Each action is represented by a function whose complexity is unpredictable, it depends on the design parameter it has to calculate. As an example, the function 'Calculate Processor Frequency()' can be very complex considering many variables: Update rate of the sensors, historical information, availability of existing central process unit (CPU), etc. 'Define CPU Architecture()', instead, could be a simple choice made by an expert designer.

\section{Implementation of the Model}

The model described by the set of diagrams presented in the previous paragraph has been used for the whole design of the satellite system, responding to the requirements stated in section 3.1. The design has 


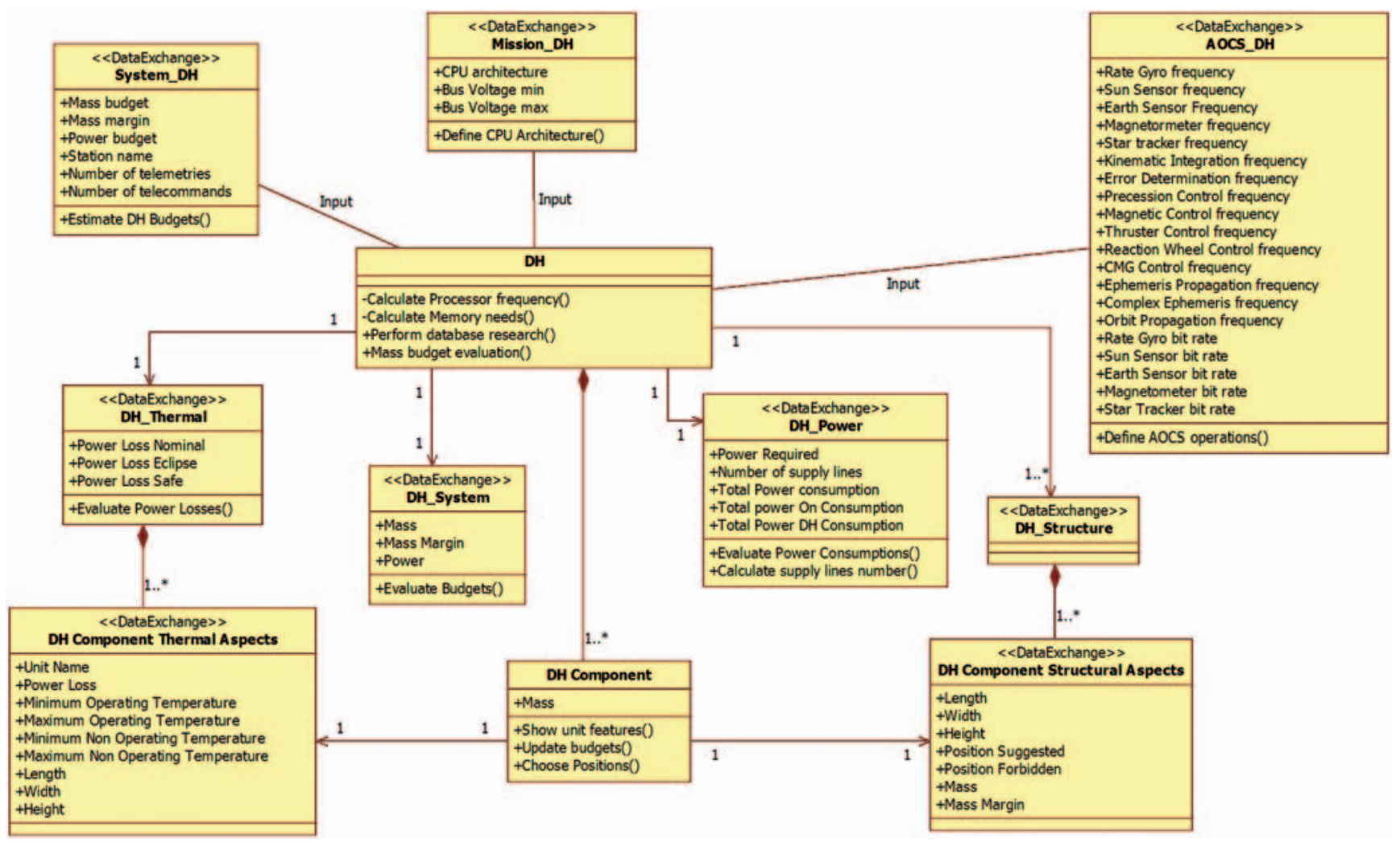

Figure 5. Data handling class diagram.



Figure 6. Data handling collaboration diagram.

followed the methodology used in ESA CDF [1, p. 2-5; 2, p. 1-3]:

- the software model developed by ESA for the exchange of data among the subsystems/engineering units

- a set of MS Excel Workbooks, one for each engineering unit, to calculate or choose the most proper values for the design parameters
Figure 7 shows the architecture of the ESA software adapted to FINGERSAT design [1, p. 6].

The MS Excel Workbooks (composed by inputs, outputs, calculation, and presentation sheets) were created by ESA for the design of each subsystem. Some of them are independent from the current project and can potentially be reused for other case studies (propulsion, data handling, system, etc). 


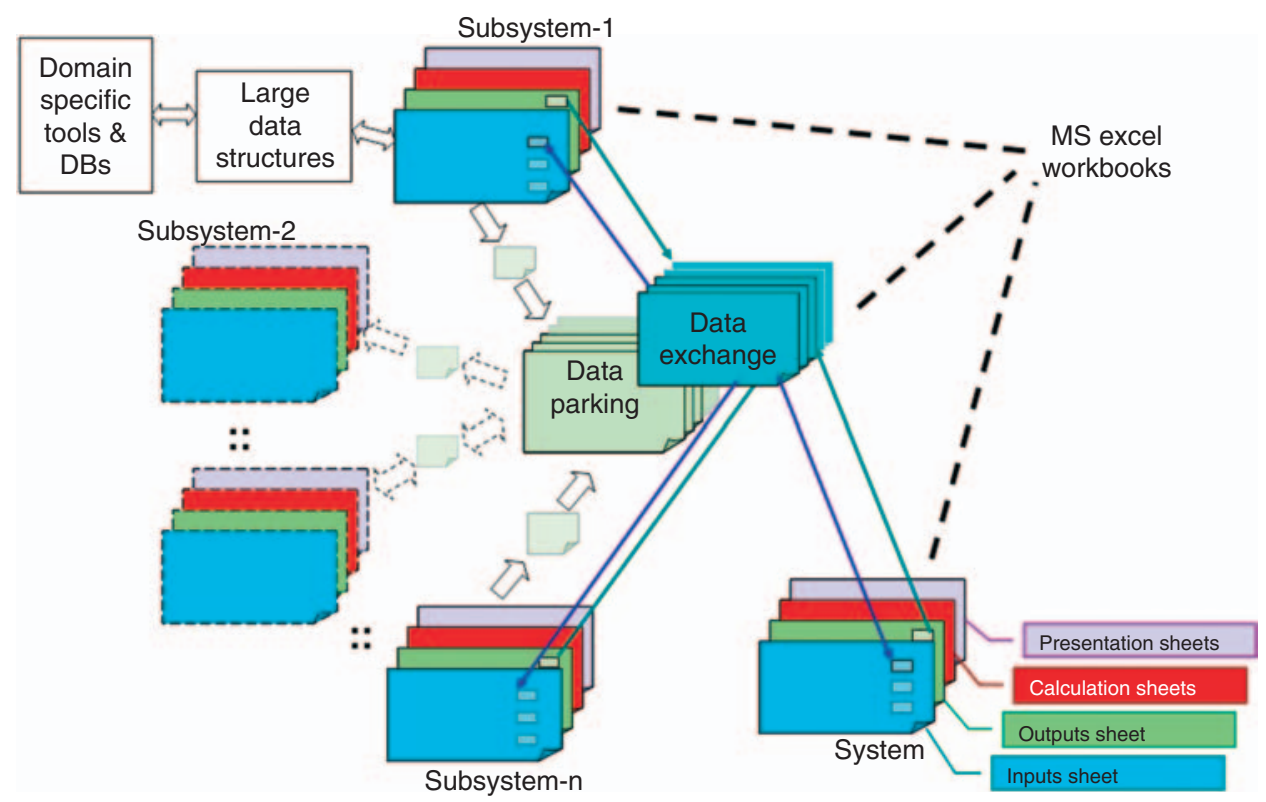

Figure 7. The architecture of European Space Agency Concurrent Design Facility software.

The choice of correct values for the design parameters is supported by the presence of a database of components in each workbook. Then each engineering unit was able to find the components necessary for the subsystem it was designing. The database presents an amount of several elements with different properties, allowing a definition of the real value of the design parameters, not only a theoretical one.

During the process the diagrams presented in the previous section constituted a roadmap for:

- the system engineering unit managing the interactions among the subsystems;

- all other engineering groups giving the complete overview of the interfaces of data exchanges from the subsystem to all the others.

Besides, the presence of activity diagrams explains the logic used during the subsystem design as depicted in the example presented in the previous paragraph.

The results of the design phases are described deeper in the teamwork report presented in partial fulfillment of the requirements for the Master degree in 'Satellites and Orbiting Platforms' of Sapienza, Università di Roma, (year 2005/06) [7].

\section{Conclusions}

Spacecraft design is a typical concurrent design activity where quite different disciplines cooperate to achieve a composite final aim. In the present work, based on ESA development in the field, a possible concurrent design procedure for the preliminary design of spacecraft has been developed, implemented and tested, producing results in the following areas:

- Analysis of dimensioning criteria and identification of design parameters for an earth observation SAR satellite (case study) and for all subsystems.

- Definition of the relations among subsystems: exchange of design parameters.

- Documentation of the static model of the design procedure using a series of UML class diagrams (described in section 4).

- Implementation of the criteria in Workbooks, programmed ad hoc for the case study using Microsoft ${ }^{\circledR}$ Office Excel (Copyright (C) 1985-2003 Microsoft Corporation).

- Documentation of the interactions among the Workbooks and description of the principal functions with the use of UML dynamic diagrams (collaboration and activity).

- Creation and loading of a database to finalize the design procedure with real components.

This article emphasizes the two documentation phases which give a formal description of data exchange, both static and dynamic, put into practice applying the presented UML model.

An entire definition of all the information needed to complete a pre-Phase A study is achieved using the presented UML model. The design phases of a satellite system, from phase A (preliminary) to phase D (detailed) design, are well described in terms of the set of requirements pertaining to each phase. The European space standards (ECSS) documents contain this piece of information. The level of detail required for phase $\mathrm{A}$ is compatible with the 
system description used in the frame of the present study.

One of the most important result of the implementation of concurrent engineering procedure in the real practice is the reduction of time-to-market by means of the reduction of design time. In order to achieve this results in the present case, a key issue is a clear description of the design variables of the space system as a whole and in terms of all its subsystems and the set up of the relevant design procedures used in the design process. In a complex system as an EO satellite, these procedures touch in most cases different areas of expertise, while in some cases the sizing of a component only refers to a specific discipline. The class diagrams illustrated in the article allow to properly describe the attribution of the design variables to the proper areas of expertise, in such a way preparing the way for setting up effective design procedure either among different areas of expertise or in the context of a single competence. The design model set up in this way will shorten the design time by limiting the design iteration and by making evident, since the beginning of the design process, all the conceptual links that are present in the different areas of expertise of the design. This will allow each member of the design team, with a specific skill, to coordinate his effort in a welldefined teamwork design process.

In fact, with a class diagram, a member of a $C D$ team completely knows what data are of interest for his subsystem and whoever is concerned with that. With an activity diagram, as well, he knows what actions to do for exchanging the aforementioned data (an action can be a software procedure, a bibliographical research, a market analysis, a Matlab routine, a meeting with other CD team members, etc.). Finally, a detailed description of these actions is achieved using activity diagrams, one for each action.

Moreover, even if the model presented is tailored to the FINGERSAT case study, the generality of the procedure is assured by the use of:

- A set of class diagram giving the static photography of the data exchange giving an answer to the questions: what each subsystem has to share with others and what can receive from the others.

- A set of Collaboration diagrams to describe the active interaction among subsystems (what happens during CDF sessions).

- An optional set of Activity diagrams which document the actions undertaken to exchange data (in FINGERSAT case these are the functions used in the workbooks).

The presented model definitively is able to provide a multidisciplinary and concurrent design environment for spacecraft design, as demonstrated by the diagrams shown in the previous pages which document with the same approach quite heterogeneous subsystems: space mission analysis, satellite structure, SAR payload, data handling subsystem. Its use is then naturally linked with a teamwork effort of design with a widespread scenario of competences well represented in the members of the team and cannot be used as a standalone one-man-only design effort. Moreover, during the practical CDF sessions, the presence of these diagrams gave to team coordinator and to each member of the team a huge capability to solve very rapidly (with only one diagram!) the outcome of inter-teams communication lacks, rapidly identifying which data are to be exchanged. This improves the team management effort and will lead to a reduced timeto-market.

The model is also very useful for educational and training purposes in space system engineering both in the academic and at the industrial environment.

\section{Acknowledgments}

The authors are indebted to Massimo Bandecchi who is responsible for ESA CDF, for his support during the development of the present study, and for leeing available to the Università di Roma La Sapienza for the purposes of research and education, and for some basic kernel models of the procedures developed by ESA. The present study was part of a team effort of all the classses of the fourth edition of the Master in Satelliti of La Sapienza, the members of which are gratefully acknowledged: Rosaria Barca, Alessandro Cricenti, Salvatore D'Addio, Federico Letterio, Daniela Pilotti, Silvia Sabatini, Daniele Scaranari, Claudio Scotognella, Stefano Serva, Manuela Sternativo, Valerio Tarantini. Also the authors are indebted with the members of all the previous classes of the Master, and in particular to Massimiliano Di Pace and Giovanni Falcucci, who formalized the study performed in the third edition of the Master.

\section{References}

1. Bandecchi, M., Melton, B. and Ongaro, F. (1999). Concurrent Engineering Applied to Space Mission Assessment and Design, Published in ESA Bulletin Nr. 99, September.

2. Bandecchi, M., Melton, B. and Gardini, B. (2000). The ESA/ ESTEC Concurrent Design Facility, EuSEC2000 September.

3. Bacchetti, M., Garutti, A. and Haines, J.E. (2001). Spacecraft Electrical Power Subsystem Design Utilising the ESA-ESTEC Concurrent Design Facility, IEEE.

4. Vergeest, J.S.M. and Horváth, I. (1999). Design Model Sharing in Concurrent Engineering: Theory and Practice, Concurrent Engineering, 7(2): 105-113.

5. Prasad, B., Morenc, R.S. and Rangan, R.M. (1993). Information Management for Concurrent Engineering: Research Issues, Concurrent Engineering, 1(1): 3-20. 
6. (Gary) Chen, S.-J. (2005). An Integrated Methodological Framework for Project Task Coordination and Team Organization in Concurrent Engineering, Concurrent Engineering, 13(3): 185-197.

7. Larson, W.J. and Wertz, J.R. (2005). Space Mission Analysis and Design, 3rd edn, Chapters 9,10, Microcosm Press and Kluwer Academic Publishers.

8. ECSS Secretariat ESA-ESTEC (1996). 'ECSS-M-30A', Requirements \& Standards Division Noordwijk, pp. 23-28, The Netherlands, 19 April.

9. Master course in 'Satellite and orbiting platforms' team (2006). CDF Technical Report - FINGERSAT Project, Università di Roma La Sapienza, April.

10. Fowler, M. (2003). UML Distilled: A Brief Guide to the Standard Object Modeling Language, 3rd edn, Chapters 1, 3, Addison Wesley.

11. Rumbaugh, J., Jacobson, I. and Booch, G. (1999). The Unified Modelling Language Reference Manual, Chapters 3,4,7,8, Addison Wesley.

\section{Davide Di Domizio}



Davide Di Domizio was born on July 12, 1974, in Italy. He earned a degree in Computer Science engineering at the University 'Federico II' of Naples (1998), a Master Course in 'Systems Design' at University 'RomaTre' of Rome (2003) and a Master Course in 'Satellites and Orbiting Platforms' at Sapienza, Università di Roma (2006). Until 2005, he was the database administrator and a software project manager at the Italian Air Force Logistic Information Center. Since 2005, he has been teaching the 'Oracle Database Applications' course at Italian National Reasearch Council (CNR). He attended the regular courses for officers at Italian Air Force Academy (19931998), and Captain since 2001. He is now employed in Italian Air Staff dealing with Italian Defence Spatial Programmes, test ranges, and UAVs.

\section{Paolo Gaudenzi}



Paolo Gaudenzi is full professor of aerospace structures and director of the Master course in Satellites and orbiting platforms at Sapienza, Università di Roma. He is author of more than 100 papers, 35 of which published in international refereed journals and is member of numerous journals editorial boards and scientific committees. His main research interest cover space systems, aerospace structures, smart structures, computational mechanics, and cost engineering. He was responsible of research projects funded by Italian and European bodies like ESA, the Italian Ministry for University, and research and by private companies. 